\title{
Speaker Independent Vowel Recognition using Backpropagation Neural Network on Master-Slave Architecture
}

\author{
J.V.S. Srinivas, \\ DVRCET, Hyderabad.
}

\author{
G. Sandhya Prafulla \\ DRKIST, Hyderabad.
}

\author{
P. Premchand, PhD. \\ Prof., OU,UCE, Hyderabad.
}

\begin{abstract}
Objective of the work is speaker independent recognition of vowels of British English. Back propagation is one of the simplest and most widely used methods for supervised training of multi layer neural networks. In this paper we use parallel implementation of Backpropagation (BP) on Master Slave architecture to recognize speaker independent eleven steady state vowels of British English. We perform the recognition task on both sequential and parallel implementation. The performance parameters speed-up, optimal number of processors and processing time are evaluated for both implementations.
\end{abstract}

\section{General Terms}

Vowel dataset from UCI, Backpropagation algorithm, Parallel implementation, Master-Slave architecture, Learning rate.

\section{INTRODUCTION}

Speech recognition is the ability of a machine or program to identify words and phrases in spoken language and convert them to a machine-readable format. The first serious speech recognizer was developed in 1952 by Davis, Biddulph, and Balashek of Bell Labs. The machine takes a human utterance as an input and returns a string of words, phrases or continuous speech in the form of text as output. The conventional methods of speech recognition insist in representing each word by its feature vector and pattern matching with the statistically available vectors using HMM or neural network [1], [2]. In this work we develop a neural network model that learns the system in least possible time and that could recognize the speaker independent utterances even though the utterance is with noise.

Connectionist Bench (Vowel Recognition - Deterding Data) Data Set from UCI Machine learning repository is used to test our recognition system based on Backpropagation Neural Network that was implemented parallel on Master-Slave Architecture. The dataset consists of a three dimensional array: vowel data [speaker, vowel, input]. The speakers are indexed by integers 0-89. (Actually, there are fifteen individual speakers, each saying each vowel six times.) The vowels are indexed by integers $0-10$. For each utterance, there are ten floating-point input values, with array indices 0-9. The data from "speakers" $0-47$ is taken to train the network and data from remaining speakers $48-89$ for testing purpose [3],[4].

.Backpropagation (BP) is one of the widely used ANN models, though it takes a long time for learning some applications [5]. A lot of research is being carried out to reduce the learning time of $\mathrm{BP}$. One of the approaches to minimize the learning time is parallel implementing the algorithm on multiple systems [6]. Implementations on commercially available dedicated parallel machines were developed. The Backpropagation Network (BPN) can be parallelized by partitioning the number of patterns, or by partitioning the network or by combination. Several researchers have developed parallel algorithms for different types of parallel computers like transputers, systolic arrays, multiple bus system, and hypercube, for diverse range of applications [7-10]. As the cost of dedicated parallel machines is high, existing equipment in standard computer labs is found to be economical alternative for a wide range of engineering applications. The most important feature is that these resources can be shared with the other applications that require them.

In our model we have mapped each neuron of the network to a processor so that the parallel computer becomes a physical model of the network. The proposed parallel implementation avoids re-computation of weights and requires less communication cycle per pattern. The communication of data among the processors in the computing network is also less. We obtain the performance parameters like speed-up, optimal number of processors and processing time for both sequential implementation and parallel implementation on Master Slave architecture.

\section{MATHEMATICAL MODEL}

In this paper, we consider a BPN with three layers $(l=0,1,2)$ having Nl neurons in each layer. We trained the network by using both sequential and parallel algorithms and respective implementations of time are discussed in the following sections.

\subsection{Sequential Backpropagation Algorithm}

The BP algorithm [5] is a supervised learning algorithm, and is used to find suitable weights, such that for a given input pattern (X0), the network output (Y2i) should match with the target output (ti). The algorithm is divided into two phases, namely, forward phase, error back propagation phase. Error backpropagation phase includes weight updates also. The details on each of these phases and the time taken to process are discussed below.

Step 0: Initialize weights (set to small random values)

Step 1: While stopping condition is false do steps 2-9

Step 2: For each training pair do steps 3-8 


\section{Feed forward}

Step 3 : For each input unit $x k=y k 0 \quad(k=1, \ldots . . N 0)$ input signal $\mathrm{xk}$ and broadcast this signal to all units in the layer above(the hidden units)

Step 4: Each hidden unit yj1 $(\mathrm{j}=1,2 \ldots \mathrm{N} 1)$ sums its weighted input signals and context signals. Then applies the activation function to compute its output signal.

$\mathrm{yj} 1(\mathrm{t})=\mathrm{f}\left(\sum_{\mathrm{k}=0}^{\mathrm{N} 0} \mathrm{wjk} 1 \mathrm{yk} 0\right)$

Here $y 01=1$ and $w j 01$ is the bias and send this signal to all units in the layer above.

Step 5 : Each output unit yi2 $(i=1,2 \ldots \ldots N 2)$ sums its weighted input signals and applies the activation function to compute its output signal.

$$
\mathrm{yi} 2=\mathrm{f}\left(\sum_{\mathrm{j}=0}^{\mathrm{N} 1} w \mathrm{wij} 2 \mathrm{yj} 1\right)
$$

calculates its weight correction terms

$\Delta w i j 2=\eta \delta i 2$ yj1 $\mathrm{j}=1,2, \ldots \mathrm{N} 1$

and sends $\delta \mathrm{i} 2 \mathrm{i}=1,2, \ldots \mathrm{N} 2$ to the units in the layer below.

Step 7: Each hidden unit yj2 (i =1,2..N1) sums its delta inputs from units in the layer above to find corresponding error information term

$$
\begin{gathered}
\delta \mathrm{j} 1=\mathrm{f} 1() \sum_{\mathrm{i}=1}^{\mathrm{N} 2} \text { wij2 } \delta \mathrm{i} 2 \quad \mathrm{j}=1,2, \ldots \mathrm{N} 1 \\
\text { calculates its weight correction terms }
\end{gathered}
$$

$\Delta w j k 2=\eta \delta j 2$ yk0 where $\mathrm{j}=1,2, \ldots \mathrm{N} 1, \quad \mathrm{k}=1, \ldots \mathrm{N} 0$

Step 8: Each output unit yi2 ( $\mathrm{i}=1,2, \ldots \mathrm{N} 2)$ updates its weights

$$
w i j 2=w i j 2+\eta \delta i 2 \text { yj1 } \quad j=1,2, \ldots \ldots . . . N 1
$$

Similarly each hidden unit yj1 $(\mathrm{j}=1,2, \ldots \mathrm{N} 1)$ updates its weights

$w j k 1=w j k 1+\eta \delta j 2$ yk0 $\quad \mathrm{k}=1,2, \ldots \ldots \ldots$ N0

$$
\begin{aligned}
& \text { Step } 9 \text { : } \text { Error matrix } E=[E 1, E 2, \ldots E p] \\
& \text { where Ei[ei1, ei2 } \ldots, \text { eiN2] } \\
& \text { Calculate } E 2=\frac{1}{2} \sum_{i=1}^{\mathrm{N} 2} \sum_{j=1}^{\mathrm{p}} \text { eij2 . }
\end{aligned}
$$

If $\mathrm{E} 2<$ Threshold vale, store wjk1 \& wij2 and

stop

else go to step 2.

Let tm, ta, and tac be time taken for one floating point multiplication, addition, and calculation of activation value respectively. The time taken to complete the forward phase (T1) is given by

$\mathrm{T} 1=\mathrm{N} 1(\mathrm{~N} 0+\mathrm{N} 2) \mathrm{M}+(\mathrm{N} 1+\mathrm{N} 2)$ tac where $\mathrm{M}=\mathrm{ta}+\mathrm{tm}$

The time taken to complete the error back propagation phase is represented by $\mathrm{T} 2$ and is calculated as

$\mathrm{T} 2=(1+\mathrm{N} 1) \mathrm{N} 2 \mathrm{M}+(\mathrm{N} 1+\mathrm{N} 2)$ tac Back propagation

Step 6 : Each output unit yi2 $(\mathrm{i}=1,2 \ldots \ldots \ldots \mathrm{N} 2)$ receives a target pattern corresponding to the input training pattern and computes error ei and error information term $\delta \mathrm{i} 2$

$$
\begin{aligned}
& \text { ei=(yi2-ti) and } \delta i 2=\text { ei f } 1() \text { where } i=1,2, \ldots N 2 \\
& i=1,2, \ldots . . . N 2 ; y 01=1 \text { and } \text { wi02 is the bias. }
\end{aligned}
$$

The time taken to update the weight matrix between the three layers is represented by $\mathrm{T} 3$ and it is equal to

$$
\mathrm{T} 3=\mathrm{N} 1(\mathrm{~N} 0+\mathrm{N} 2) \mathrm{M}+(\mathrm{N} 0+\mathrm{N} 2) \mathrm{N} 1 \mathrm{tac}
$$

Let tac $=\beta \mathrm{M}$. The total processing time (Tseq) for training a single pattern in one iteration is the sum of the time taken to process the three phases and is given as

Tseq $=\mathrm{T} 1+\mathrm{T} 2+\mathrm{T} 3=(\mathrm{N} 1 \mathrm{~K}+\mathrm{N} 2 \gamma) \mathrm{M}+\mathrm{N} 1(\mathrm{~N} 0+\mathrm{N} 2)$ tac Where $\mathrm{k}=2 \mathrm{~N} 0+3 \mathrm{~N} 2+2 \beta$ and $\gamma=1+2 \beta$

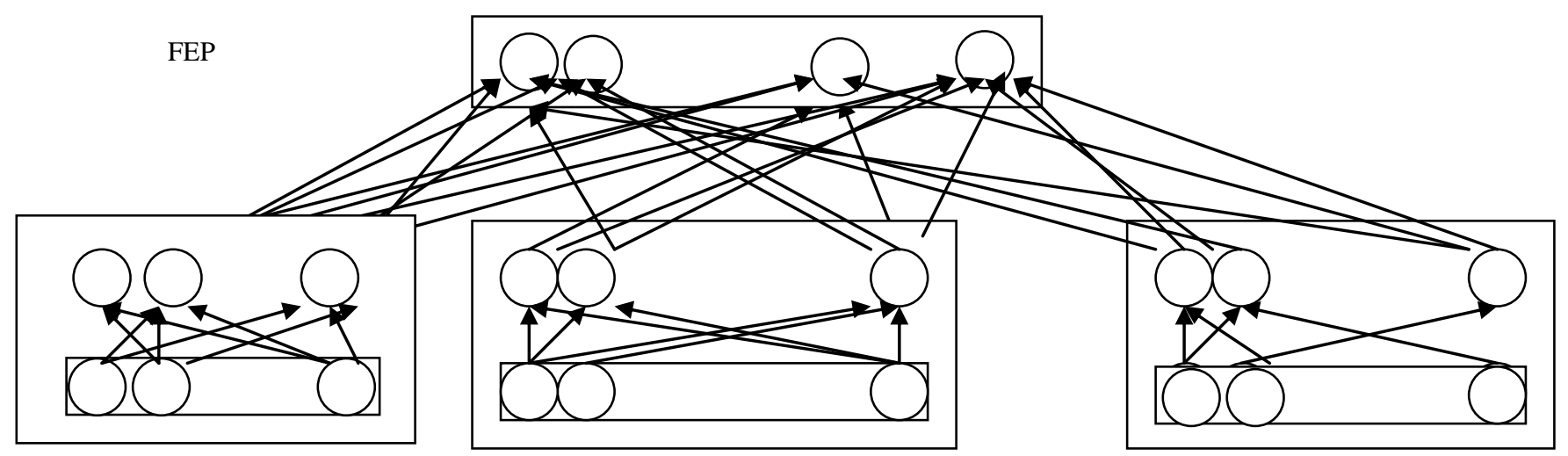

Fig 1: Slave Processors

\subsection{Parallel Implementation}

In parallel implementation, the hidden layer is partitioned using vertical parallelism and weight connections are partitioned on the basis of synaptic level parallelism. The Master Slave architecture of the MLP network used in the proposed scheme is shown in the Fig.1. In this architecture there is one front - end processor (FEP) and ' $\mathrm{m}$ ' slave processors. The output layer is placed on front - end processor, hidden layer is partitioned into $\mathrm{N}_{1} / \mathrm{m}$ neurons and the partitions are placed on slave processors. The input neurons are placed on all the slave processors [6].

In this architecture the communication is only between the FEP and $\mathrm{m}$ Slave processors. Each of the Slave processor with FEP executes three phases of BP training algorithm. Parallel execution of the three phases and the corresponding processing time for each phases are calculated. 


\section{Algorithm for Front End Processor (FEP):}

Step 0 : Download all the input vectors onto all the processors.

$$
\mathrm{E}_{\mathrm{old}}=\text { LARGE_VALUE. }
$$

Step 1 : while stopping condition is false do steps $1-7$

Step 2: For each training pair with old weights do

steps 3-5

Step 3: Receive input partial sums calculated at processors. Store the hidden layer outputs in the context layer.

Step 4: Compute the output vector $\mathrm{O}$ of neurons in the output layer, error $\mathrm{E}_{\text {new }}$ and error information terms $\delta_{\mathrm{i}}{ }^{1}$ and $\delta_{\mathrm{i}}{ }^{2}$

Step 5 : Send this information to nodes.

Step 6: If $\left(E_{\text {new }}^{2}<E_{\text {old }}^{2}\right)$

Send the command "Realize weights" to nodes. $\mathrm{E}_{\text {old }}=\mathrm{E}_{\text {new }}$

else

nodes.

Send "Discard new weights" command to

Step 7: If $\left(E_{\text {new }}^{2}<\right.$ Threshold value $)$

Send terminate command to the nodes.

else

Send iterate command and go to Step2

\section{$\underline{\text { Algorithm for nodes: }}$}

Step 0: Initialize the weights (set to small random values).

Receive all input vectors from FEP.

Step 1: While stop signal is not received from FEP do steps 2-

Step 2: For each training pair do steps 3-8

Feed forward:

Step 3: Each input unit $\left(\mathrm{x}_{\mathrm{i}}=\mathrm{y}_{\mathrm{i}}{ }^{0}, \mathrm{i}=1,2, \ldots . . \mathrm{N}_{0}\right)$ receives input signal $\mathrm{x}_{\mathrm{i}}$ and broad casts this signal to all units in the layer above.
Step 4: Each hidden unit $\left(\mathrm{y}_{\mathrm{i}}{ }^{1} \mathrm{i}=1 \ldots \ldots . \mathrm{N}_{1}\right)$ present on this processor sums its weighted input signals and applies its activation function.

$$
\mathrm{y}_{\mathrm{i}}{ }^{1}=\mathrm{f}\left(\sum_{\mathrm{j}=0}^{\mathrm{N} 0} \mathrm{w}_{\mathrm{ij}}{ }^{1} \mathrm{y}_{\mathrm{j}}{ }^{0}\right)
$$

and sends this signal to all units in the layer above.

Step 5: Each output unit $\mathrm{y}_{\mathrm{i}}^{2}\left(\mathrm{i}=1 \ldots . . \mathrm{N}_{2}\right)$ sums its partial weighted input signals

$$
\mathrm{y}_{\mathrm{i}-\mathrm{in}}^{2}=\sum_{\mathrm{j}=1}^{\mathrm{N} 1 / \mathrm{m}} \mathrm{w}_{\mathrm{ij}}{ }^{2} \mathrm{y}_{\mathrm{j}}^{1}
$$

Where $\mathrm{i}=1,2, \ldots \mathrm{N}_{2}$ and $\mathrm{m}$ is the number of nodes. $\mathrm{N}_{1} / \mathrm{m}$ neurons of the hidden layer are distributed onto $\mathrm{m}$ processors.

Send the partial sums to FEP

Error backpropagation:

Step 6: Receive $\mathrm{E}_{\text {old }}, \delta_{\mathrm{i}}{ }^{1}$ and $\delta_{\mathrm{i}}{ }^{2}$ from FEP and compute $\Delta \mathrm{w}$ and $\Delta \mathrm{w}^{2}$

$$
\begin{aligned}
& \text { Step 7: } \mathrm{w}_{\mathrm{ij}}{ }^{2} \text { old }=\mathrm{w}_{\mathrm{ij}}{ }^{2} ; \mathrm{w}_{\mathrm{jk}}{ }^{1} \text { old }=\mathrm{w}_{\mathrm{jk}}{ }^{1} \\
& \mathrm{w}_{\mathrm{ij}}{ }^{2}=\mathrm{w}_{\mathrm{ij}}{ }^{2}+\sum_{\mathrm{m}=1}^{\mathrm{p}} \Delta \mathrm{w}_{\mathrm{ij}}{ }^{2}(\mathrm{~m}) \\
& \mathrm{i}=1,2, \ldots \mathrm{N}_{2}, \mathrm{j}=1,2, \ldots \mathrm{N}_{1} \\
& \mathrm{w}_{\mathrm{jk}}{ }^{1}=\mathrm{w}_{\mathrm{jk}}{ }^{1}+\sum_{\mathrm{m}=1}^{\mathrm{p}} \Delta \mathrm{w}_{\mathrm{jk}}{ }^{1}(\mathrm{~m}) \mathrm{j}=1,2, \ldots \mathrm{N}_{1}, \mathrm{k}=1,2, \ldots \mathrm{N}_{0}
\end{aligned}
$$

Step 8: Receive commands from FEP

If (cmd= Realize weights) $\eta=\eta^{*} 1.2$

If $(\mathrm{cmd}=$ discard weights $)$

$\left\{\mathrm{w}_{\mathrm{jk}}{ }^{1}=\mathrm{w}_{\mathrm{jk}}{ }^{1}\right.$ old $; \mathrm{w}_{\mathrm{ij}}{ }^{2}=\mathrm{w}_{\mathrm{ij}}^{2}$ old $;$ Set $\eta=$ initial value $\}$

Step 9: If $(\mathrm{cmd}=$ Terminate $)$

Save the weights $\mathrm{w}^{1}$ old and $\mathrm{w}^{2}{ }_{\text {old }}$

$$
\text { elseif ( } \mathrm{cmd}=\text { iterate) go to Step 2; }
$$

The approximate time taken to process the forward phase $\left(\mathrm{T}_{1}\right)$ is given by.

$\mathrm{T}_{1}=\left\{\left(\mathrm{N}_{0}+\mathrm{N}_{2}\right) \mathrm{N}_{1} \mathrm{M}\right\} / \mathrm{m}+\left[\left(\mathrm{N}_{1} / \mathrm{m}\right)+\mathrm{N}_{2}\right] \mathrm{t}_{\mathrm{ac}}+\mathrm{N}_{2} \mathrm{mt}_{\mathrm{a}}+\mathrm{t}_{\mathrm{com}}^{1}$ where $M=t_{a}+t_{m}$ 
The approximate time taken to calculate error at the FEP and at the slave processors is

$\mathrm{T}_{2}=\left(\mathrm{N}_{1}+\mathrm{m}\right) \mathrm{N}_{2} \mathrm{M} / \mathrm{m}+\left\{\mathrm{N}_{1} / \mathrm{m}+\mathrm{N}_{2}\right\} \mathrm{t}_{\mathrm{ac}}+\mathrm{t}_{\mathrm{com}}$

The processing time to update the weight connections $\left(\mathrm{T}_{3}\right)$ is given by

$\mathrm{T}_{3}=\left(\mathrm{N}_{\mathrm{o}}+\mathrm{N}_{2}\right) \mathrm{N}_{1} \mathrm{M} / \mathrm{m}+\left(\left(\mathrm{N}_{\mathrm{o}}+\mathrm{N}_{2}\right) \mathrm{N}_{1} / \mathrm{m}\right) \mathrm{t}_{\mathrm{m}}$

Let $\mathrm{T}_{\mathrm{M}}$ be the time taken to train a single pattern in proposed HP scheme, and it is equal to the algebraic sum of time taken to process all the three phases.

$\mathrm{T}_{\mathrm{P}}=\mathrm{M}\left[2\left(\mathrm{~N}_{0}+\mathrm{N}_{2}\right) \mathrm{N}_{1}+\left(\mathrm{N}_{1}+\mathrm{m}\right) \mathrm{N}_{2}\right] / \mathrm{m}+\left[\left(\mathrm{N}_{1} / \mathrm{m}\right)+\mathrm{N}_{2}\right] \mathrm{t}_{\mathrm{ac}}$ $+\left(\mathrm{N}_{\mathrm{o}}+\mathrm{N}_{2}\right) \mathrm{N}_{1} \mathrm{t}_{\mathrm{m}} / \mathrm{m}+\mathrm{M} \log \mathrm{m}\left\{2 \sigma+\mu\left[\left(\mathrm{N}_{1} / \mathrm{m}\right)+\mathrm{N}_{2}\right]\right\}$

Here $t_{\text {com }}=\log p\left[T_{\text {ini }}+T_{c} D\right]$ where $p$ is the number of processors, $D$ is data size( number of words), $T_{\text {ini }}$ is startup time and $\mathrm{T}_{\mathrm{c}}$ is per word transfer time.[11]

\subsection{Analytical Performance Comparison}

\subsubsection{Speed up analysis}

Speed-up for m-processor system is the ratio between the time taken by uniprocessor to the time taken by parallel algorithm in $\mathrm{m}$-processor network.

The speed-up ratio for parallel implementation can be formulated as

$$
\begin{aligned}
\mathrm{S}= & \mathrm{T}_{\mathrm{seq}} / \mathrm{T}_{\mathrm{P}} \\
= & {\left[\left(\mathrm{N}_{1} \mathrm{k}+\mathrm{N}_{2} \beta\right) \mathrm{M}+\mathrm{N}_{1}\left(\mathrm{~N}_{0}+\mathrm{N}_{2}\right) \mathrm{t}_{\mathrm{ac}}\right]\left\{\mathrm { M } \left[2\left(\mathrm{~N}_{0}+\mathrm{N}_{2}\right) \mathrm{N}_{1}\right.\right.} \\
& \left.+\left(\mathrm{N}_{1}+\mathrm{m}\right) \mathrm{N}_{2}\right] / \mathrm{m}+\left[\left(\mathrm{N}_{1} / \mathrm{m}\right)+\mathrm{N}_{2}\right] \mathrm{t}_{\mathrm{ac}}+\left(\mathrm{N}_{\mathrm{o}}+\mathrm{N}_{2}\right) \mathrm{N}_{1} \mathrm{t}_{\mathrm{m}} / \\
\mathrm{m} & \left.+\mathrm{M} \log \mathrm{m}\left\{2 \sigma+\mu\left[\left(\mathrm{N}_{1} / \mathrm{m}\right)+\mathrm{N}_{2}\right]\right\}\right\}^{-1}
\end{aligned}
$$

If the network size is extremely larger than the number of processors $\mathrm{m}$, then the speed up ratio will approach $\mathrm{m}$. This is due to extra computation required in weight update phase and extra communication in exchanging the hidden neurons activation values.

\subsubsection{Optimal number of processors}

We observe from TP that the increase in the number of processors will lead to increase in the communication time and decrement in the computation time. The total processing time will decrease first and then increase after a certain number of processors. So, there exists an optimal number of processors $\mathrm{m} *$ for which processing time is minimum.

\subsubsection{Difference between processing times}

From the expressions of Tseq and TP the difference in time processing is calculated as follows.

$\mathrm{T}_{\text {seq }}-\mathrm{T}_{\mathrm{P}}=\left[\left(\mathrm{N}_{1} \mathrm{k}+\mathrm{N}_{2} \beta\right) \mathrm{M}+\mathrm{N}_{1}\left(\mathrm{~N}_{0}+\mathrm{N}_{2}\right) \mathrm{t}_{\mathrm{ac}}\right]-\left\{\mathrm{M}\left[2\left(\mathrm{~N}_{0}+\right.\right.\right.$ $\left.\left.\mathrm{N}_{2}\right) \mathrm{N}_{1}+\left(\mathrm{N}_{1}+\mathrm{m}\right) \mathrm{N}_{2}\right] / \mathrm{m}+\left[\left(\mathrm{N}_{1} / \mathrm{m}\right)+\mathrm{N}_{2}\right] \mathrm{t}_{\mathrm{ac}}+\left(\mathrm{N}_{\mathrm{o}}+\mathrm{N}_{2}\right) \mathrm{N}_{1}$ $\left.\mathrm{t}_{\mathrm{m}} / \mathrm{m}+\mathrm{M} \log \mathrm{m}\left\{2 \sigma+\mu\left[\left(\mathrm{N}_{1} / \mathrm{m}\right)+\mathrm{N}_{2}\right]\right\}\right\}$

Assume that $\mathrm{N}_{1}=\mathrm{N}_{2}=\mathrm{N}_{3}=\mathrm{m}=\mathrm{N}$. Then

$\mathrm{T}_{\mathrm{seq}}-\mathrm{T}_{\mathrm{P}} \cong$

$\left[5 \mathrm{~N}^{2}-5 \mathrm{~N}(\sigma+\mu)-7+\beta\left(2 \mathrm{~N}^{2}+4 \mathrm{~N}-4\right)\right] \mathrm{t}_{\mathrm{a}}+\left[5 \mathrm{~N}^{2}+\mathrm{N}-5 \mathrm{~N}(\sigma\right.$

$\left.+\mu)-10+\beta\left(2 \mathrm{~N}^{2}+4 \mathrm{~N}-4\right)\right] \mathrm{t}_{\mathrm{m}}>0$
From the above equation it is clear that the time taken by parallel implementation is less than the time taken by the sequential algorithm.

\section{EXPERIMENTAL \& STUDY RESULTS}

The eleven steady state vowels of British English in the dataset are i , O, I, C:,E,U:,A,u.a:, 3: Y. The speech signals were low pass filtered at $4.7 \mathrm{kHz}$ and then digitized to 12 bits with a $10 \mathrm{kHz}$ sampling rate. Twelfth order linear predictive analysis was carried out on six 512 sample Hamming windowed segments from the steady part of the vowel. The reflection coefficients were used to calculate $10 \mathrm{log}$ area parameters, giving a 10 dimensional input space. We trained the letter recognition dataset on the BPN with 10 input neurons, 8 hidden neurons and 3 output neurons. There was a substantial decrease in the learning time of the networks in parallel implementation. The results are shown graphically in fig- 2 .

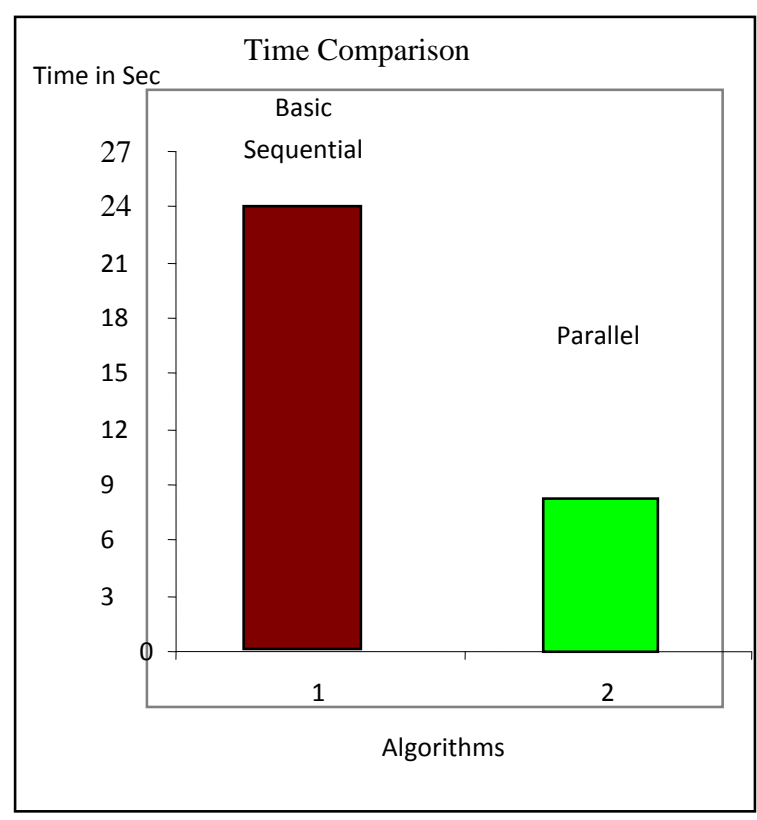

Fig-2 Learning Rate:0.2

We have also compared the learning performance by varying the number of processors. We observed that after a certain stage there is no advantage with increment in the number of processors. The optimal number of processors in our example is 9 as shown in Fig 3. 


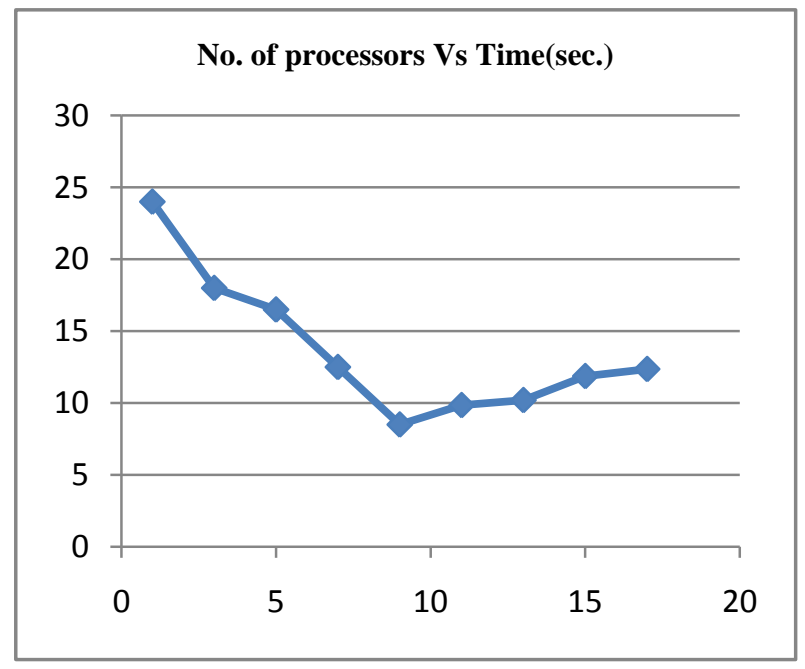

Fig 3

\section{CONCLUSIONS}

In this paper, we implemented a parallel BP algorithm on a cluster of computers connected over Ethernet LAN to recognize the vowel dataset presented. The neural network is mapped onto Master-Slave architecture. The analytical performance of the proposed algorithm is compared with the sequential counterpart. Using the hybrid-partitioning scheme, re-computation of weights is avoided and the communication time is reduced. As one hidden layer is adequate for a large number of applications, in the present project work the algorithm is developed for neural nets with one hidden layer.

\section{ACKNOWLEDGEMENTS}

We acknowledge UCI machine learning repository for giving the opportunity to use the Connectionist Bench (Vowel Recognition - Deterding Data) Data Set to train and test our model.

\section{REFERENCES}

[1] L. R. Rabiner, B. H. Juang, "Fundamentals of Speech Recognition", Prentice Hall, Englewood Cliffs, New Jersey, 1993.

[2] L.Deng and D.O'Shaughnessy, "Speech processing: a dynamic and optimization-oriented approach", Marcel Dekker Inc.,2003

[3] Frank, A. \& Asuncion, A. (2010). UCI Machine Learning Repository [http://archive.ics.uci.edu/ml]. Irvine, CA: University of California, School of Information and Computer Science.

[4] UCI repository of machine learning databases.

[5] S. Haykins, Neural Networks_A Comprehensive Foundation. PEA, 2004.

[6] Srinivas JVS, Bhogendra Rao PVRR, Kamakshi Prasad V. "Parallel Implementation of Backpropagation on Master Slave Architecture" Volume: 1, On Page(s): 217 - 221 International Conference on Computational intelligence and Multimedia Applications 2007

[7] V. Kumar, S. Shekar, and M.B. Amin, "A Scalable Parallel Formulation of the Back-Propagation Algorithm for Hypercubes and Related Architectures," IEEE Trans. Parallel and Distributed Systems, vol.5, no.10,pp.10731090, Oct. 1994.

[8] Czauderna, T.; Seiffert, U.: Implementation of MLPnetworks running backpropagation on various parallel computer hardware using MPI. Proceedings of the $5^{\text {th }}$ International Conference on Recent Advances in Soft Computing (RASC), Nottingham, United Kingdom, December 16-18, 2004, pp.116- 121. [ISBN 1842331108]

[9] Han-wook Lee and Chan-ik, An Efficient Parallel Block Backpropagationn Learning Algorithm in TransputerBased Mesh- Connected Parallel Computers, IEICE Trans. Inf. \& Syst., vol. E83-D, No.8, August, 2000.

[10] Faramarz valafar and Okan K. Ersoy, Parallel Implementation of Back-Propagation Neural Network on MASPAR MP-1

[11] Annanth Grama, Anshul Gupta, George Karypis, Vipin Kumar - Introduction to parallel computing, PEA, 2004. 